\section{Current status of ultrasonography of the finger}

\author{
Seun Ah Lee', Baek Hyun Kim', Seon-Jeong Kim², Ji Na Kim³, Sun-Young Park', \\ Kyunghee $\mathrm{Choi}^{5}$
}

\begin{abstract}
${ }^{1}$ Department of Radiology, Korea University Ansan Hospital, Korea University College of Medicine, Ansan; ²Department of Radiology, Myongji Hospital, Seonam University College of Medicine, Goyang; ${ }^{3}$ Department of Radiology, Chungnam National University Hospital, Chungnam National University School of Medicine, Daejeon; ${ }^{4}$ Department of Radiology, Hallym University Sacred Heart Hospital, Hallym University College of Medicine, Anyang; ${ }^{5}$ Department of Radiology, Incheon Baek Hospital, Incheon, Korea
\end{abstract}

The recent development of advanced high-resolution transducers has enabled the fast, easy, and dynamic ultrasonographic evaluation of small, superficial structures such as the finger. In order to best exploit these advances, it is important to understand the normal anatomy and the basic pathologies of the finger, as exemplified by the following conditions involving the dorsal, volar, and lateral sections of the finger: sagittal band injuries, mallet finger, and Boutonnière deformity (dorsal aspect); flexor tendon tears, trigger finger, and volar plate injuries (volar aspect); gamekeeper's thumb (Stener lesions) and other collateral ligament tears (lateral aspect); and other lesions. This review provides a basis for understanding the ultrasonography of the finger and will therefore be useful for radiologists.

Keywords: Anatomy; Fingers; Ultrasonography; Upper extremity

\section{Introduction}

The finger is a very small and complex structure, which makes it difficult to evaluate using imaging modalities. Although ultrasonography (US) has several disadvantages, including a small focused field of view, operator-dependency, and limitations in visualizing deep joints and cartilage, recent developments in advanced high-resolution US have improved the ability of US to visualize very small superficial structures, such as the finger [1]. The use of high-frequency linear or L-shaped hockey stick probes (10 MHz or more) allows rapid assessments, static and dynamic evaluations (including flexion and extension movements), and stress studies of the finger, and real-time scanning can be used during clinical examinations $[1,2]$. These advances have made it necessary to acquire a specific understanding of finger anatomy and related sonographic findings. The purpose of this review is to familiarize radiologists with the normal anatomy of the finger and its correlation with sonograms. We also review several disorders involving the soft tissues of the finger, based on their locations within the finger (dorsal, volar, and lateral regions), and provide detailed descriptions and illustrations.

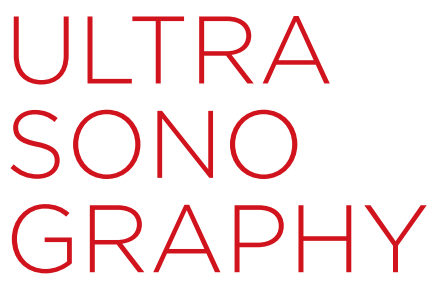

\section{REVIEW ARTICLE}

http://dx.doi.org/10.14366/usg. 15051 pISSN: 2288-5919 - elSSN: 2288-5943 Ultrasonography 2016;35:110-123

Received: September 1, 2015 Revised: November 23, 2015 Accepted: November 24, 2015

Correspondence to: Baek Hyun Kim, MD, PhD, Department of Radiology, Korea University Ansan Hospital, Korea University College of Medicine, 123 Jeokgeum-ro, Danwongu, Ansan 15355, Korea

Tel. $+82-31-412-5228$

Fax. +82-31-412-5226

E-mail: kimbaekh@hanmail.net

This is an Open Access article distributed under the terms of the Creative Commons Attribution NonCommercial License (http://creativecommons.org/ licenses/by-nc/3.0/) which permits unrestricted noncommercial use, distribution, and reproduction in any medium, provided the original work is properly cited.

Copyright @ 2016 Korean Society of Ultrasound in Medicine (KSUM)

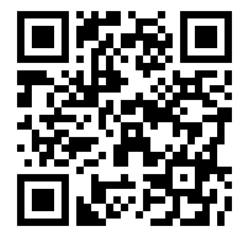

How to cite this article:

Lee SA, Kim BH, Kim SJ, Kim JN, Park SY, Choi $\mathrm{K}$. Current status of ultrasonography of the finger. Ultrasonography. 2016 Apr;35(2):110123. 


\section{Dorsal Aspect}

\section{Normal Anatomy}

The extensor hood stabilizes the extensor tendon at the metacarpophalangeal (MCP) joint, whereas the dorsal extensor apparatus stabilizes the proximal interphalangeal (PIP) joint. The extensor hood is a fibrous expansion on the dorsal aspect of the proximal phalanx (PP) of the fingers and is made up of three structures: sagittal bands, oblique fibers, and transverse fibers. The sagittal bands, which represent the main stabilizer of the extensor hood, maintain the extensor tendons in the midline of the metacarpal head during flexion and extension of the fingers. US depicts the sagittal bands as thin hyperechoic bands that join the extensor tendons at the MCP joint level (Fig. 1) [3].
The two main components of the extensor tendons are extrinsic muscles and intrinsic muscles. The extrinsic muscles, which originate from the forearm and elbow and insert into the hand, comprise the extensor digitorum communis, extensor indicis proprius, and extensor digiti quinti minimi. The intrinsic muscles, which originate and insert into the hand, include the interosseous and lumbrical muscles [4]. Distal to the MCP joint, the extensor tendon splits into a central slip and two lateral slips. The extrinsic extensor tendon continues in the central and lateral slips, the while the intrinsic extensor tendons contribute to the formation of the lateral slips [4]. After the lateral slips conjoin with the intrinsic muscles, they are termed conjoint tendons and form the terminal tendon that inserts into the dorsal aspect of the distal phalangeal base [4]. On US, the central slip can be seen up to its insertion into the middle

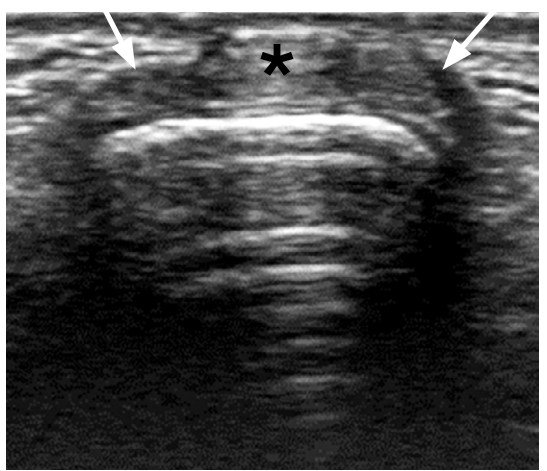

A
Fig. 1. Normal ultrasonography findings of the dorsal aspect of the finger.

A. Transverse sonogram at the metacarpophalangeal joint level shows the oblique, thin, and hyperechoic sagittal bands (arrows) that join the extensor tendons (asterisk). More distally, the tendon splits into a central slip and two lateral slips. B. Longitudinal sonogram allows accurate assessment of the dorsal aspect of the finger. The extensor tendon (arrowheads) is seen as a hypoechoic tubular structure in the subcutaneous fat layer that covers the bony cortex and the joints. Note the central tendon inserting into the dorsal aspect of the middle phalangeal base (circle) and the terminal tendon inserting into the dorsal aspect of the distal phalangeal base (dotted circle). DP, distal phalanx; MP, middle phalanx; PP, proximal phalanx.

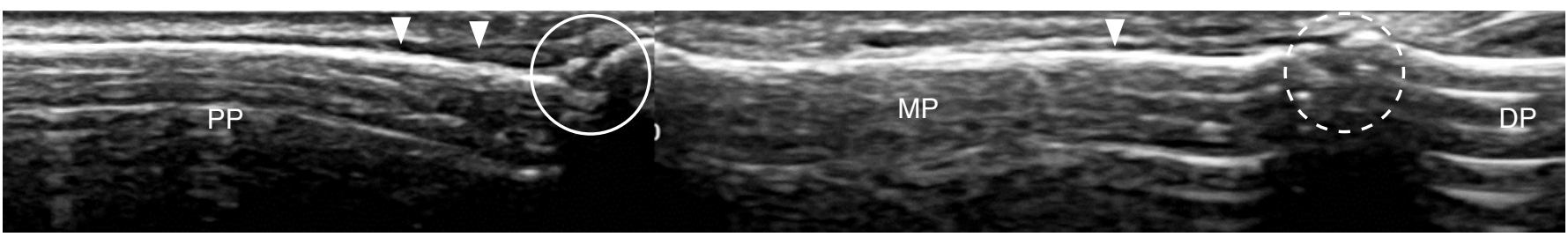

B

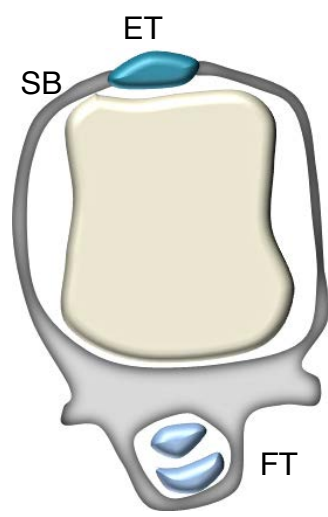

A

e-ultrasonography.org
Sagittal band tear

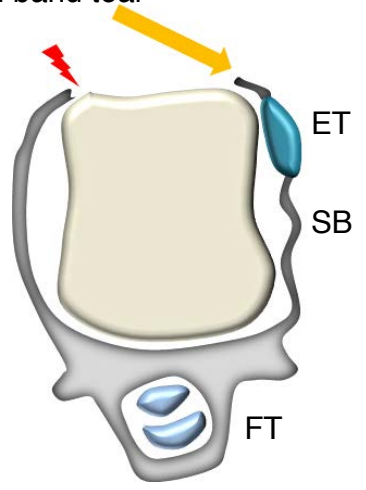

Fig. 2. Schematic images of the sagittal band and sagittal band tears.

A, B. At the metacarpophalangeal joint, the extensor tendons are stabilized by the extensor hood and particularly by the sagittal band (A). Subluxation or dislocation of the extensor tendon occurs as a result of a tear in the sagittal band (B). ET, extensor tendon; $\mathrm{FT}$, flexor tendon; SB, sagittal band.
B

$B$ 
phalangeal base in the dorsal aspect. More distally, US depicts the lateral slips as thin, flattened, hyperechoic structures close to the phalangeal cortex [2]. Since it is difficult to accurately assess the dorsal aspect of the interphalangeal (IP) joints in the transverse plane, US of joints is mainly performed in the longitudinal plane. In longitudinal US, the central slip is seen as a hyperechoic structure running through the subcutaneous soft tissue over the echogenic bony cortex, whereas the two lateral slips are hardly visible (Fig. 1) [2].

\section{Sagittal Band Injuries (Boxer's Knuckle)}

The sagittal band is the main stabilizer of the extensor tendon in the dorsal aspect of the MCP joint during flexion and extension of the fingers. Since the sagittal band prevents deviation of the extensor tendon during flexion and bowstringing during MCP joint hyperextension, injury to the sagittal band causes extensor tendon dislocation (Fig. 2, Video clip 1) [5]. Sagittal band injuries result from acute direct trauma or chronic, repetitive microtrauma, and typically occur in boxers [2]. The most frequent location of this injury is the third finger [6]. The site of the tear may affect whether the extensor tendon is displaced towards the radial or ulnar side [5]. Although the most frequent site of disruption is the radial sagittal band, such that the extensor tendon dislocates in an ulnar direction, radial subluxation can occur with forced valgus injury $[4,6]$. Since the second and fifth fingers have two tendons per finger, they may displace in different directions, one to the radial side and the other to the ulnar side [2]. US demonstrates irregular thickening of the abnormal sagittal band with hypoechogenicity (Figs. 3, 4) [2]. The extensor tendon can appear normal or can become swollen with loss of the fibrillar pattern, suggestive of a partial tear. On dynamic examination, the extensor tendon can be subluxed or dislocated during finger flexion in the transverse plane (Figs. 3, 4) [2].

\section{Extensor Tendon Tears}

Extensor tendon tears typically occur in two locations in the finger.

\section{Mallet finger}

Mallet finger, which is commonly referred to as baseball finger or cricket finger, occurs at the distal phalangeal base, which is the insertion site of the extensor tendon, also known as the terminal tendon. This injury occurs when the distal interphalangeal (DIP) joint is abruptly forced into extreme flexion, at which time the tendon may pull off a piece of bone (Fig. 5) [2,4]. The clinical symptoms of mallet finger include pain, swelling, and inability to extend the DIP

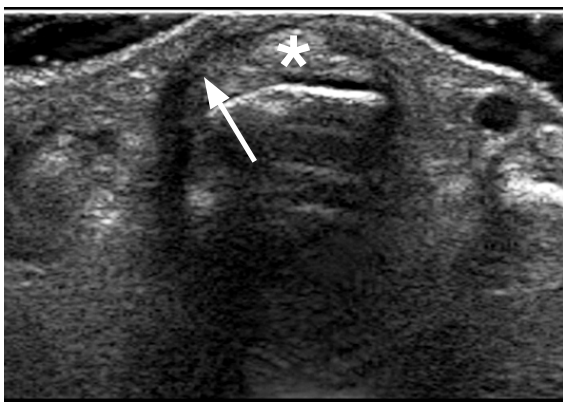

A

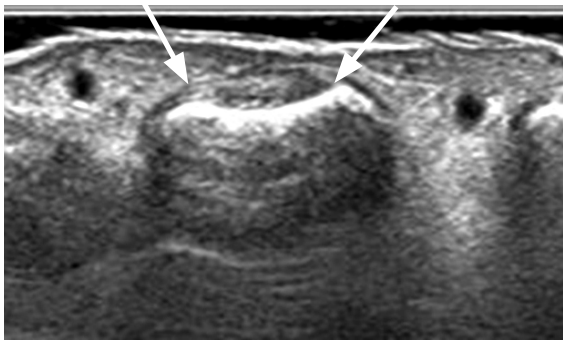

A

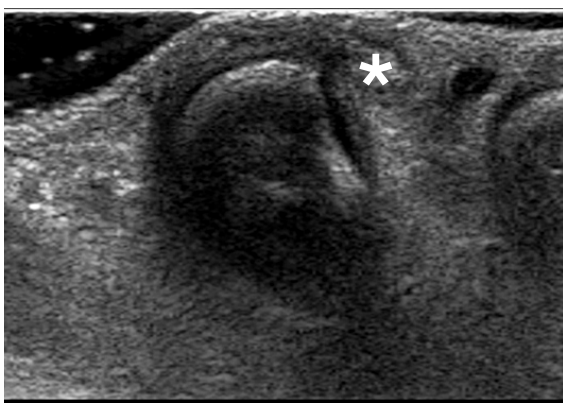

B

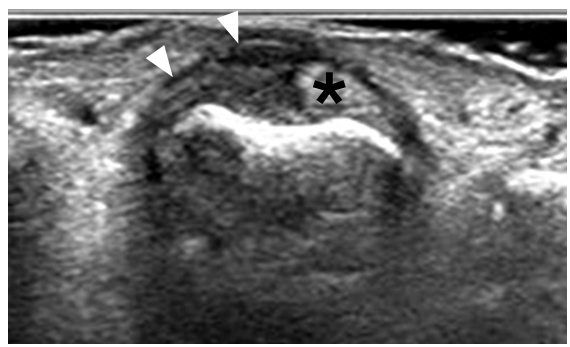

B
Fig. 3. Sagittal band tear in a 43-year-old woman.

A. A transverse sonogram of the third metacarpophalangeal joint when the finger is extended shows an abnormal radial sagittal band with irregularity and hypoechogenicity (arrow). The extensor tendon (asterisk) is positioned normally. B. A dynamic examination obtained in the transverse plane during finger flexion shows dislocation of the extensor tendon (asterisk).

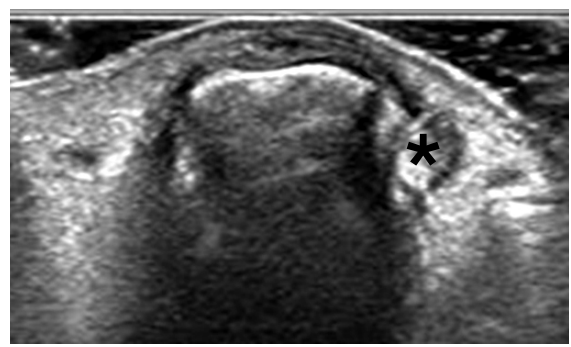

C

Fig. 4. Sagittal band tear in a 69-year-old woman.

A. Transverse sonogram of a normal third metacarpophalangeal (MCP) joint demonstrates the extensor hood (arrows) as a thin, echogenic structure that overlies the dorsal aspect of the MCP joint. B. Transverse sonogram of an abnormal third MCP joint shows an irregular, hypoechoic thickening of the sagittal band (arrowheads). The extensor tendon (asterisk) has deviated to the ulnar border of the MCP joint under extension. C. During full finger flexion, transverse sonogram shows dislocation of the extensor tendon (asterisk) on the ulnar side. 


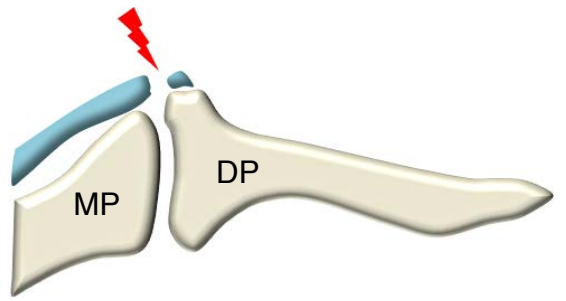

A

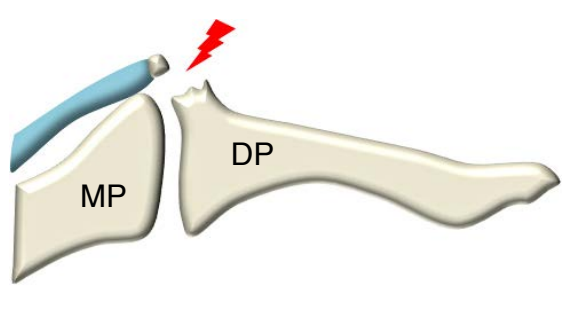

B

Fig. 5. Schematic images of mallet finger. A. Mallet finger is a tear of the extensor tendon at the insertion of the terminal tendon into the distal phalangeal base. B. The tendon may pull off a piece of bone, suggestive of an avulsion fracture. DP, distal phalanx; MP, middle phalanx.

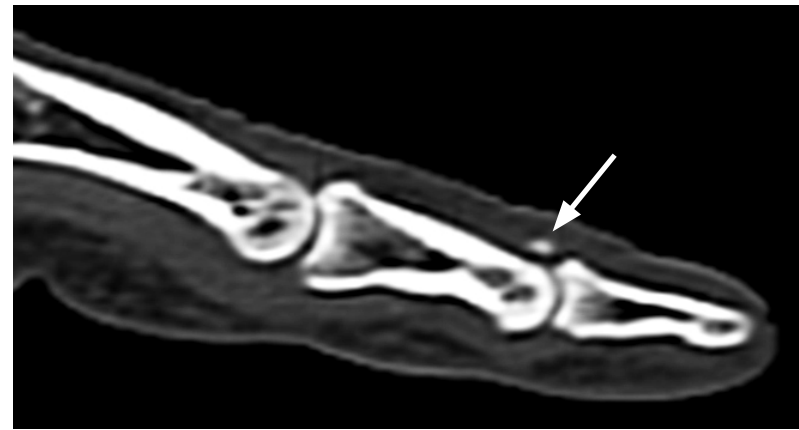

A

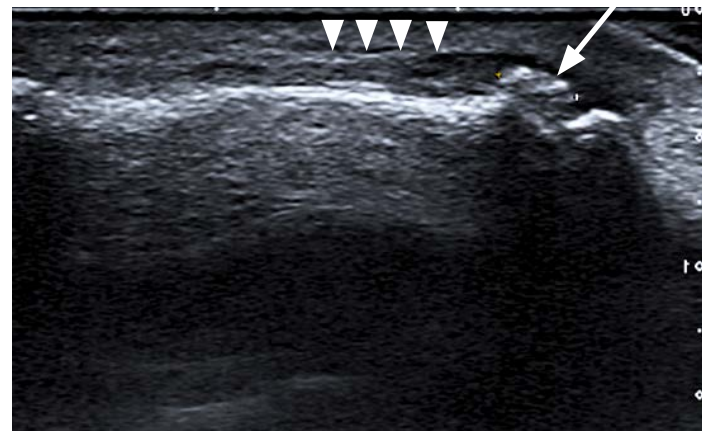

B

Fig. 6. Avulsion fracture of the terminal tendon (mallet finger) in a 64-year-old man.

A. Sagittal computed tomography scan demonstrates a tiny bone fragment (arrow) at the distal interphalangeal joint of the second finger on the dorsal side. B. Longitudinal sonogram shows the detached bone fragment (arrow) in continuity with the retracted tendon (arrowheads) and loss of substance in the distal phalangeal base.

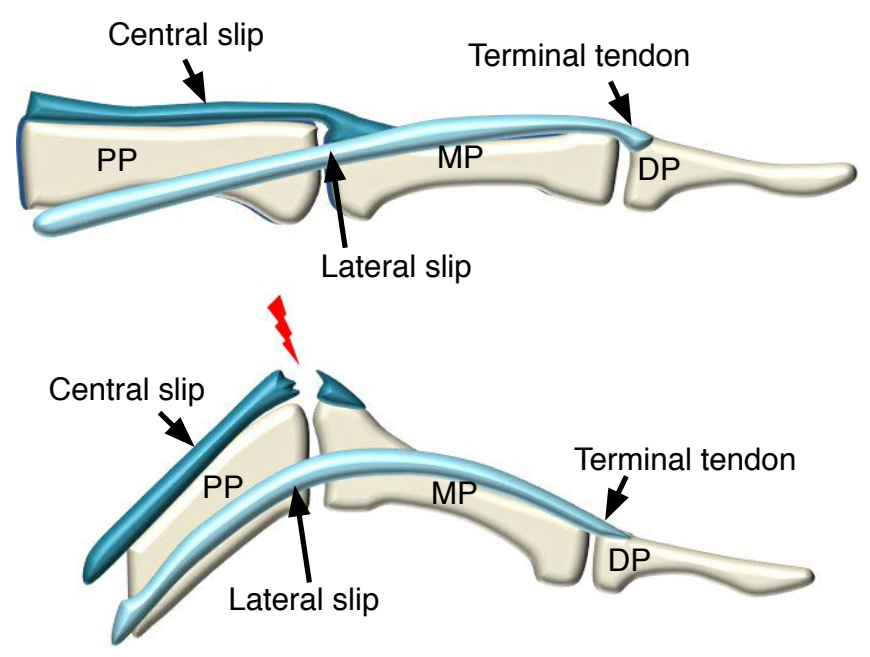

Fig. 7. Schematic images of Boutonnière deformity. Boutonnière deformity is an injury involving the central slip of the extensor tendon over the proximal interphalangeal joint. DP, distal phalanx; MP, middle phalanx; PP, proximal phalanx.

joint [4].

On US, instead of tendon echoes at the tendon insertion site of the distal phalanx, an irregular hypoechoic soft tissue lesion is usually present over the distal shaft of the middle phalanx, indicative of the retracted tendon end. In the case of an avulsion fracture, the detached bone fragment at the retracted tendon end and loss of substance in the distal phalangeal base can also be detected on US (Fig. 6) [2].

\section{Boutonnière deformity}

The second most frequent site of injuries involving the extensor tendon is at or near the central slip insertion site of the middle phalangeal base. This injury is known as Boutonnière deformity (Figs. 7, 8). The mechanisms underlying Boutonnière deformity include acute violent flexion of the PIP joint, a blow to the dorsum of the middle phalanx, or volar dislocation of the PIP joint [4]. In the acute stage, Boutonnière deformity presents with pain, swelling of the PIP joint, mild extension lag, and reduced extension strength against resistance. After the acute stage, the intact lateral slips move the volar aspect, inducing flexion of the PIP joint, an increase in the force exerted on the intact terminal tendon insertion, and subsequent extension of the DIP joint [4]. On US, the central slip of the injured extensor tendon demonstrates a lack of tendon echoes that would indicate insertion into the middle phalangeal base, whereas the lateral slips are intact on both sides of the middle phalanx (Fig. 9) [2]. 


\section{Volar Aspect}

\section{Normal Anatomy}

Two flexor tendons are present on the volar side of the fingers: the flexor digitorum profundus (FDP) and the flexor digitorum superficialis (FDS). They run within the fibrous tendon sheath. Once the FDS enters the tendon sheath, it is separated into two tendons that surround the FDP and insert into the shaft of the middle

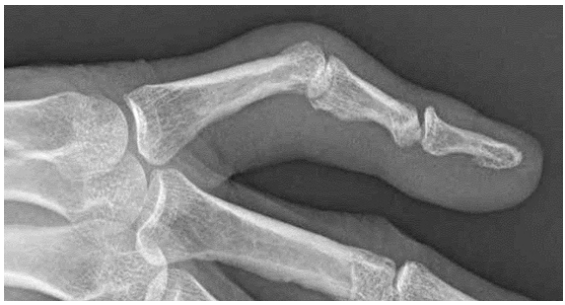

A

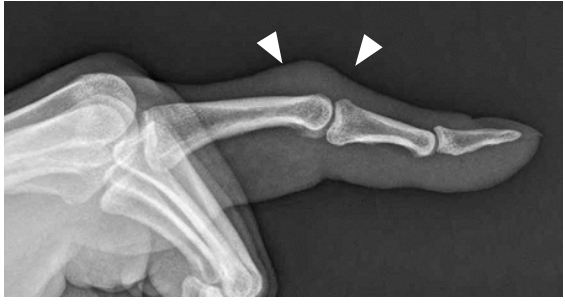

A

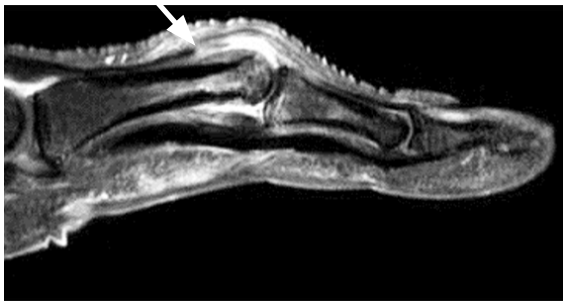

B

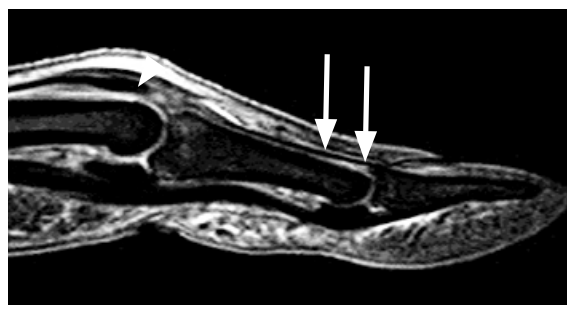

B
Fig. 8. Boutonnière deformity in a 59-yearold man.

A. Radiograph shows a classic Boutonnière deformity manifesting during flexion of the proximal interphalangeal joint and extension of the distal interphalangeal joint. B. Sagittal T2 fat saturation magnetic resonance image shows discontinuity of the central slip (arrow) at its distal insertion into the middle phalangeal base.

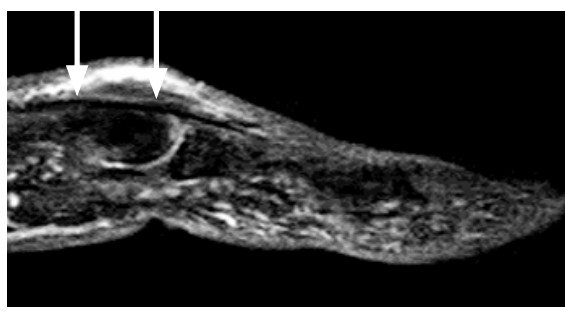

C

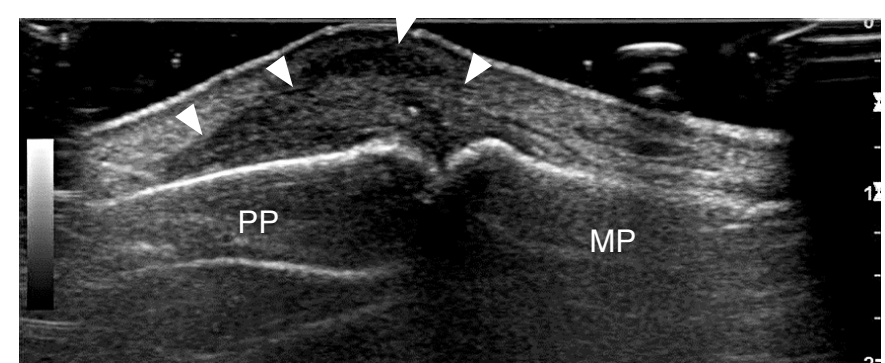

D

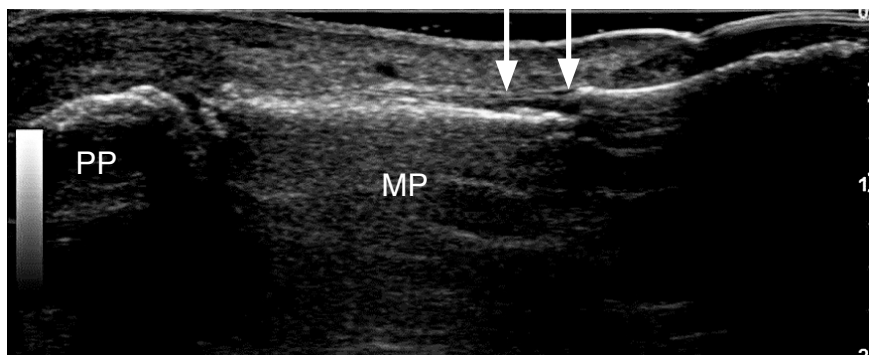

E

Fig. 9. Boutonnière deformity in a 57-year-old man.

A. Radiograph shows soft tissue swelling (arrowheads), mild flexion of the proximal interphalangeal joint, and extension of the distal interphalangeal joint. B, C. Sagittal T2 fat saturation magnetic resonance images depict complete tearing of the central slip (arrowhead), an intact lateral slip (arrows), and its insertion site (arrows) at the base of the distal phalanx. D, E. Longitudinal sonograms show a lack of tendon echoes indicating insertion into the middle phalangeal base (arrowheads), whereas the terminal tendon, composed of two lateral tendons, is inserted into the distal phalangeal base (arrows). MP, middle phalanx; PP, proximal phalanx.

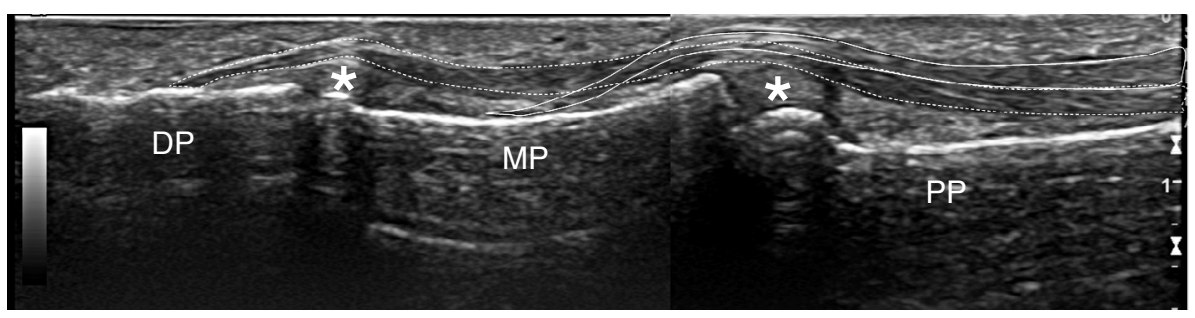

Fig. 10. Normal sonogram of the flexor tendon. A longitudinal sonogram shows the volar plates (asterisks), the flexor digitorum profundus tendon (dotted lines), its insertion into the distal phalangeal base, and the insertion of the flexor digitorum superficialis tendon (solid lines) into the middle phalanx (MP). DP, distal phalanx; $\mathrm{PP}$, proximal phalanx. 
phalanx. The FDP is initially located deep in the FDS, and then enters the cleft formed by the division of the FDS and inserts into the distal phalangeal base (Figs. 10, 11) [2].

On US, it is difficult to distinguish between the two flexor tendons (the FDP and FDS) at the level of the PIP joint; however, tilting the probe and using a dynamic study can be helpful in this regard (Fig. 12, Video clip 2) [2]. The flexor tendons can be easily assessed by dynamic examination during passive flexion of the whole finger and selective flexion of the distal phalanx. With the probe placed over the ventral aspect of the metacarpal head, US reveals gliding of both flexor tendons when the examiner passively moves both the middle and distal phalanges in flexion and extension. If the examiner moves the distal phalanx only, while locking the middle phalanx, the FDP tendon can be seen to glide under a stationary FDS (Video clip 2).

The flexor tendon sheath extends from the metacarpal neck to the DIP. A series of retinacular structures in which the sheath is thickened at five specific points, form the annular pulley system (pulleys A1-A5), while another three form the cruciate pulley system (pulleys C1-C3) (Fig. 13). These pulleys prevent excursion of the flexor tendons from the MCP and IP joints during finger flexion $[3,7]$. The A2 pulley is the thickest, strongest, and longest annular pulley, and is seen as a linear hyperechoic or hypoechoic thickening of the tendon sheath on a longitudinal scan (Fig. 14) [7]. The A1, A2, and A4 pulleys are generally visualized without any difficulty, while the A3 and A5 pulleys are inconsistently depicted. However, all pulleys can be seen on current US equipment by using higher-frequency transducers $[3,7]$.

The MCP, PIP, and DIP joints are reinforced by the volar plate, which is composed of thick, resistant fibrocartilage. The volar plates limit finger hyperextension. On longitudinal US, the fibrocartilaginous

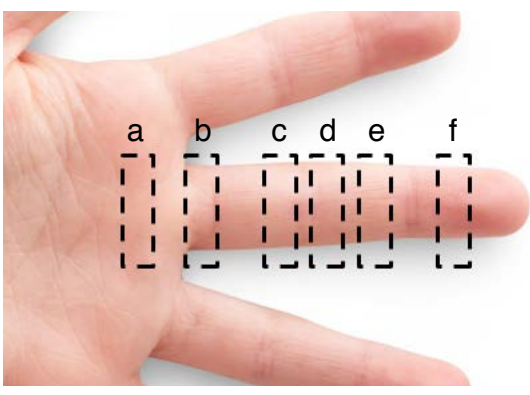

A
Fig. 11. Transverse ultrasonography of the flexor tendon.

A. Each number from a to $f$ indicates the location of the probe. B. Transverse sonograms of the flexor tendon at the corresponding probe locations demonstrate the division of the flexor digitorum superficialis (FDS) tendon into two slips that diverge to surround the flexor digitorum profundus (FDP) tendon, as well as the reunion of the FDS slips deep in the FDP. Blue color and green color indicate FDS and FDP, respectively.
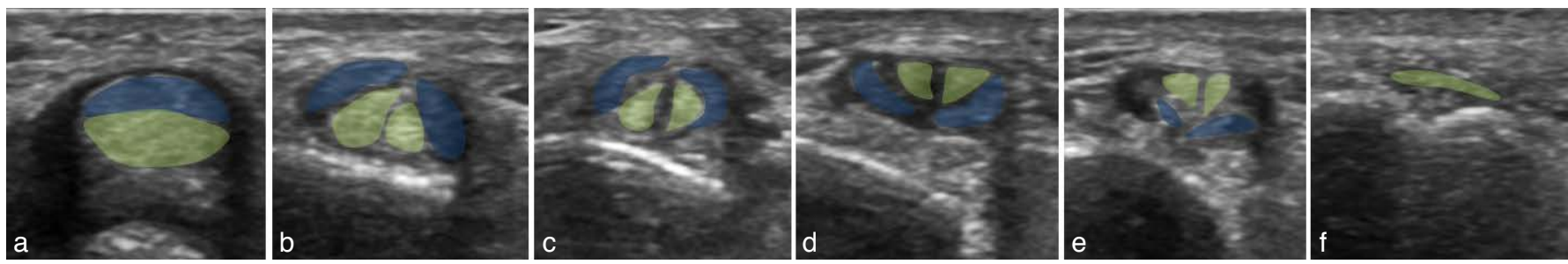

B

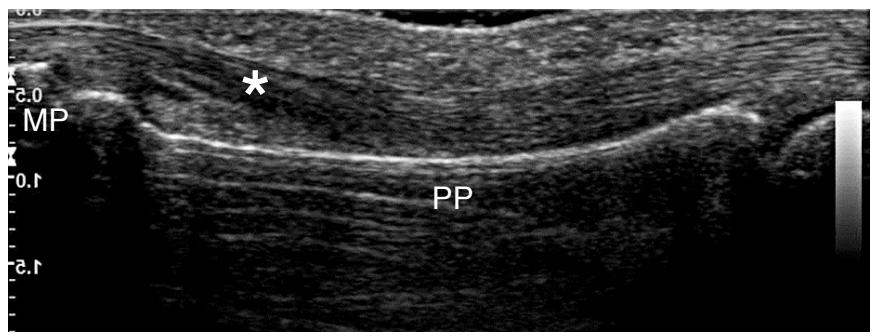

A

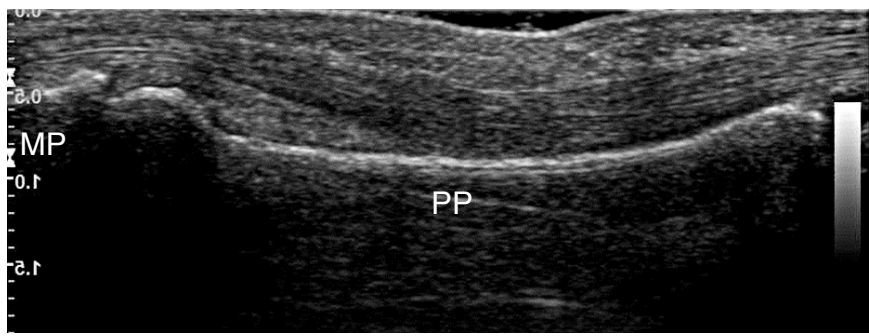

B

Fig. 12. Anisotropy of the flexor tendon.

A. Longitudinal sonogram of the flexor tendon shows an anisotropic artifact (asterisk). B. Longitudinal sonogram of the flexor tendon shows a reduced anisotropic artifact. The flexor digitorum profundus tendon sometimes displays hypoechoic artifacts caused by tendinous anisotropy. Longitudinal sonogram in precisely the same position with the transducer held parallel to the profundus tendon shows reduced anisotropic hypoechogenicity. MP, middle phalanx; PP, proximal phalanx. 


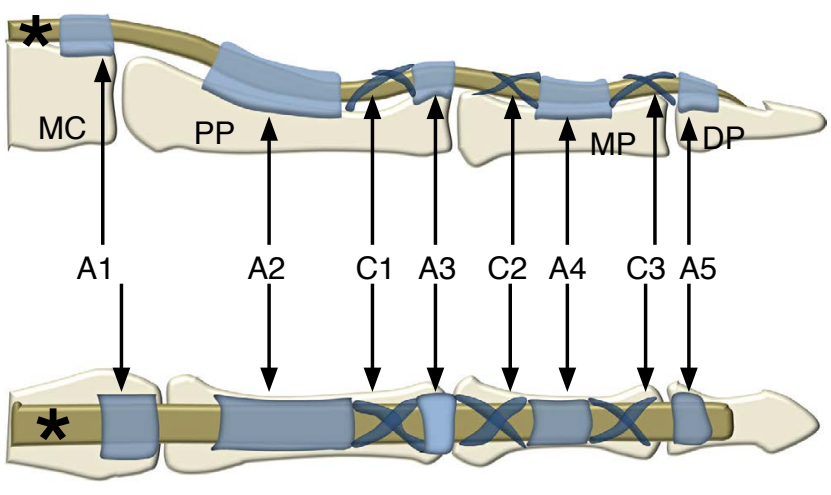

Fig. 13. Schematic image of the finger pulley system. The finger pulley system consists of five annular pulleys $(A 1-A 5)$ and three cruciform pulleys (C1-C3). MC, metacarpal bone; PP, proximal phalanx; MP, middle phalanx; DP, distal phalanx.

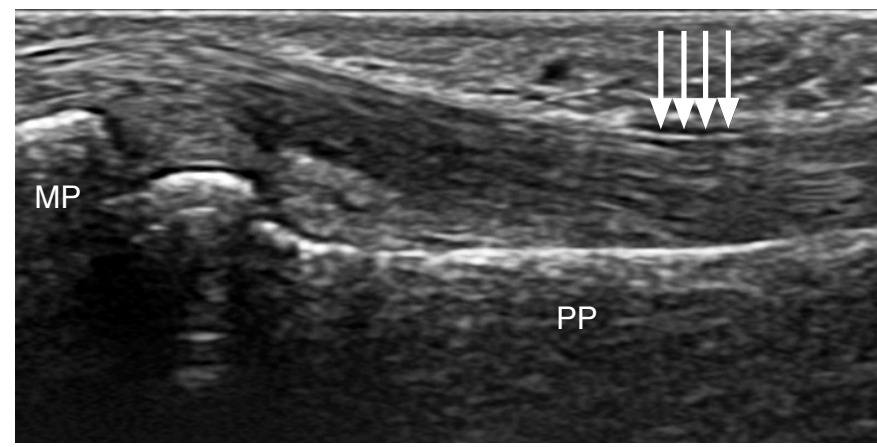

Fig. 14. Ultrasonography of a normal A2 pulley. Longitudinal sonogram demonstrates a thin, hypoechoic line (arrows) at the level of the proximal third of the proximal phalanx (PP). MP, middle phalanx. volar plate is seen as a triangular hyperechoic structure, superficial to the articular cartilage that is seen as a band-like anechoic area overlying the metacarpal head (Figs. 10, 15) [2].

\section{Flexor Digitorum Tendon Tears}

Injuries of the flexor tendons are not as common as those of the extensor tendons. Avulsion of the FDP is the most frequent type of closed injury of the flexor tendons, including the FDP and FDS $[2,4]$ Avulsion of the FDP can be caused by a forced passive hyperextension injury during active finger flexion [4]. This injury, which is usually observed among young males who participate in contact sports, including rugby and football, is commonly called sweater finger or jersey finger $[2,4]$. It may sometimes cause avulsion of a piece of bone from the palmar aspect of the distal phalanx, similarly to mallet finger [2].

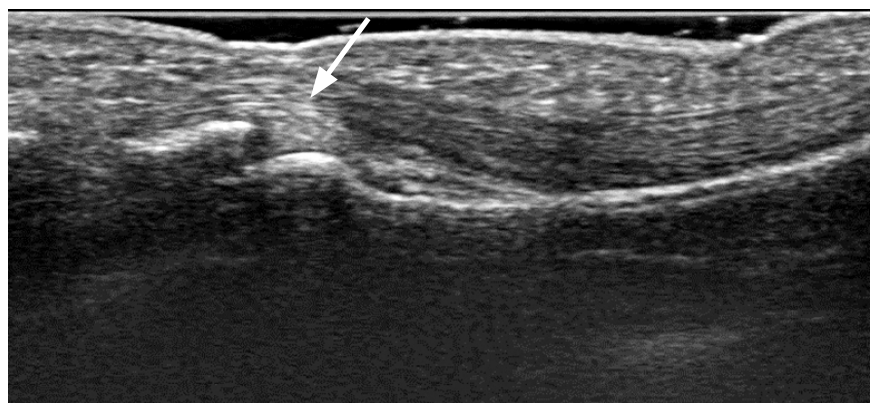

Fig. 15. Ultrasonography of a normal volar plate. Longitudinal sonogram shows thickening of the joint capsule on the volar aspect of the proximal interphalangeal joint, representing the volar plate (arrow).

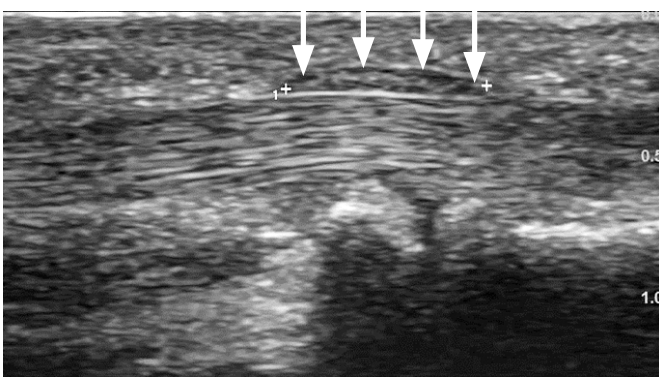

A

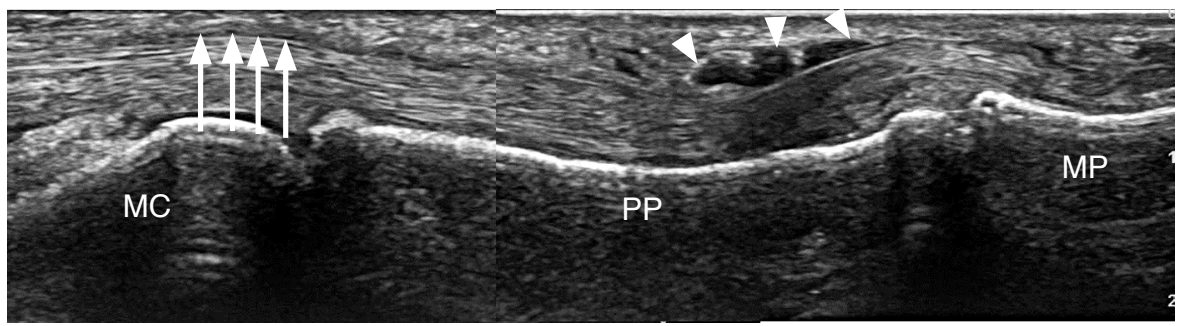

C

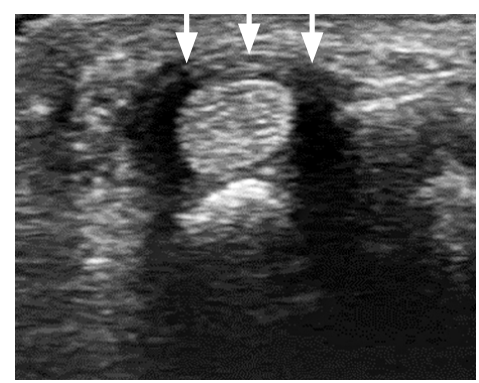

B

Fig. 16. Trigger finger in a 49-year-old woman.

A, B. Longitudinal (A) and transverse (B) sonograms of the first finger show hypoechoic thickening of the A1 pulley (arrows). The underlying flexor tendon is typically swollen and has a more rounded appearance when viewed in a cross-section. C. Longitudinal sonogram of the third finger shows the A1 pulley (arrows) with diffuse hypoechoic thickening and flexor tendon abnormalities, including fluid collection (arrowheads) along the tendon sheath. MC, metacarpal bone; MP, middle phalanx; PP, proximal phalanx. 
Dynamic US should be routinely performed during active and passive movements of the finger and during contraction against resistance in order to evaluate flexor tendon continuity and to differentiate between partial and complete tears [2]. In partial tears, US demonstrates a hypoechoic, fusiform swelling of the tendon and focal discontinuity of the internal fibrillar appearance [2]. In

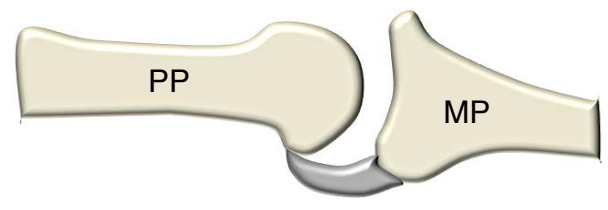

A

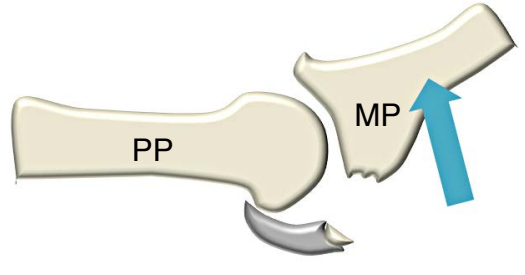

B

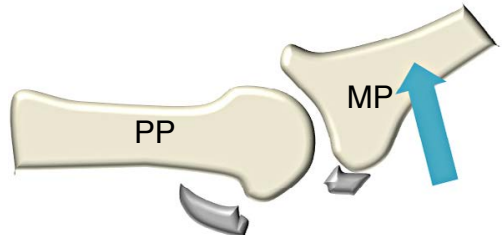

C

Fig. 17. Schematic images of volar plate injuries.

A-C. Each drawing shows normal (A), avulsion fracture (B) and substantial tear of the volar plate (C) by the hyperextension (arrows). MP, middle phalanx; PP, proximal phalanx.

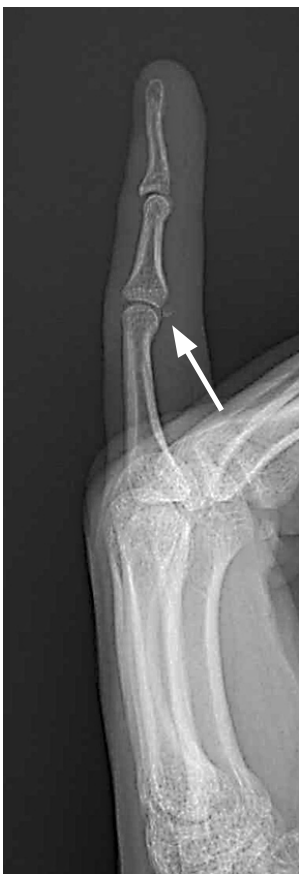

A

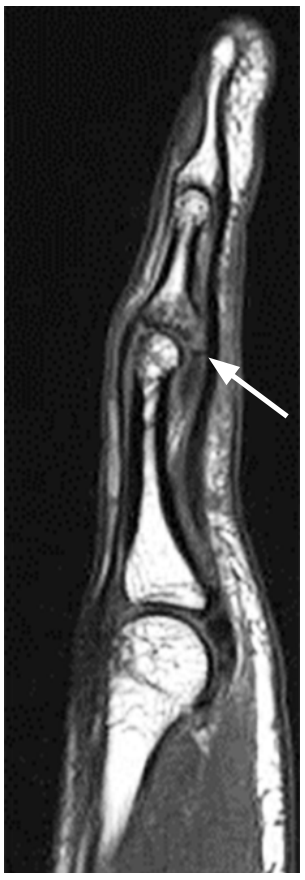

B

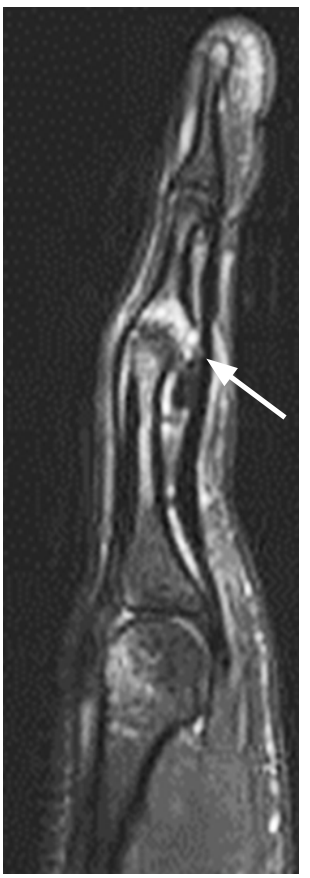

C
Fig. 18. Volar plate injury in a 21-year-old man.

A. Radiograph shows a bone fragment (arrow) at the volar aspect of the proximal interphalangeal joint. $B, C$. Sagittal T1-weighted magnetic resonance (MR) image (B) and sagittal T2 fat saturation MR image (C) demonstrate a bony fragment (arrow) detached from the phalangeal base, suggestive of an avulsion fracture of the volar plate.

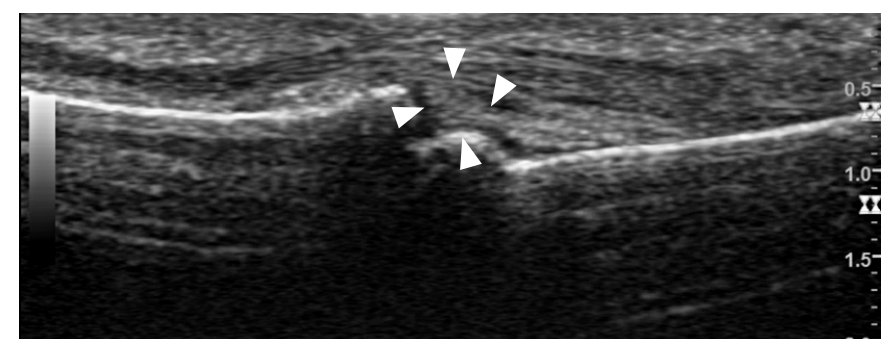

A

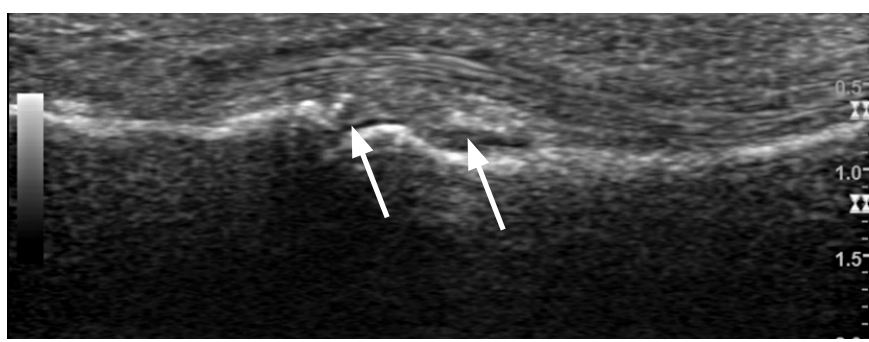

B

Fig. 19. Volar plate injury in a 14-year-old man.

A. Longitudinal sonogram of the second proximal interphalangeal (PIP) joint shows a normal volar plate (arrowheads). B. Longitudinal sonogram of the abnormal second PIP joint shows two separated fragments of the volar plate (arrows), suggestive of a volar plate tear. 
complete tears, the ruptured tendon is not seen at the injury site and the end of the frayed retracted tendon presents as an irregular and hypoechoic lesion with posterior acoustic shadowing related to sound beam refraction [2].

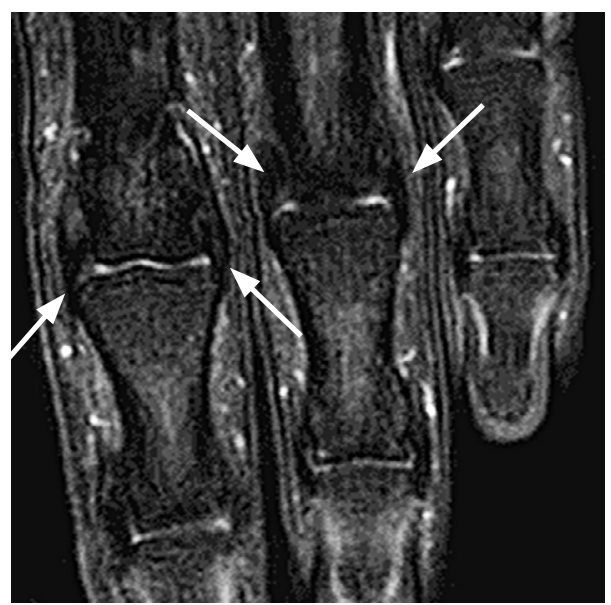

Fig. 20. Magnetic resonance (MR) image of the collateral ligament of the proximal interphalangeal (PIP) joints. Coronal T2 fat saturation MR image shows the normal collateral ligaments (arrows) as a low signal intensity band at the PIP joints. Collateral ligaments are usually best visualized in the coronal plane.

\section{Trigger Finger}

Trigger finger is a transient locking of the finger during flexion with a painful snapping sensation during extension [2]. The major hypothesis for its etiology is that it is caused by stenosing tenosynovitis of the flexor tendons with thickening of the A1 pulley [8].

US findings may show diffuse hypoechoic thickening of the A1 pulley and underlying flexor tendon abnormalities suggestive of tenosynovitis. The affected tendons are typically swollen and appear rounder in cross-sectional views under the thickened pulley than the other fingers. In addition, dynamic US can visualize the locking and snapping of the flexor tendon at the MCP level (Fig. 16, Video clip 3) $[2,8]$.

\section{Volar Plate Injuries}

Volar plate injuries are mostly caused by hyperextension of the joint and can involve avulsion fractures or substantial tears (Fig. 17). Avulsion fracture of the volar plate presents as a bony fragment detached from the phalangeal base, without tearing of the volar plate itself (Fig. 17B). These bone avulsions are commonly seen on lateral radiography and US (Fig. 18). Substantial tears of the volar plate are rarer (Fig. 17C). On US, a hypoechoic cleft within a swollen volar plate can be regarded as a sign of rupture (Fig. 19) [2].

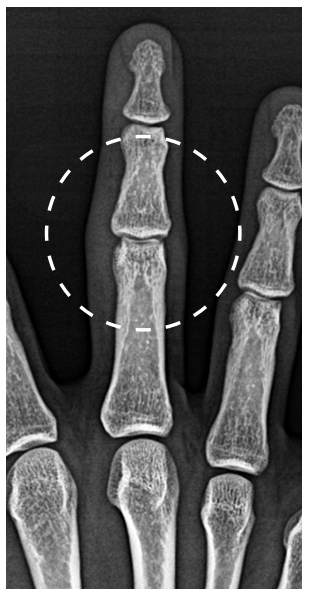

A

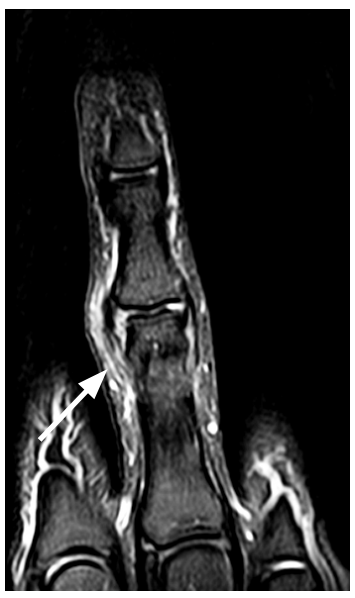

B

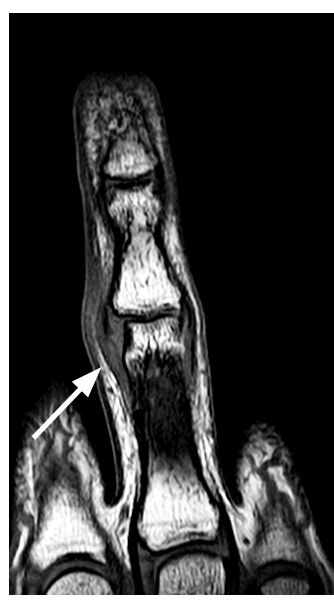

C

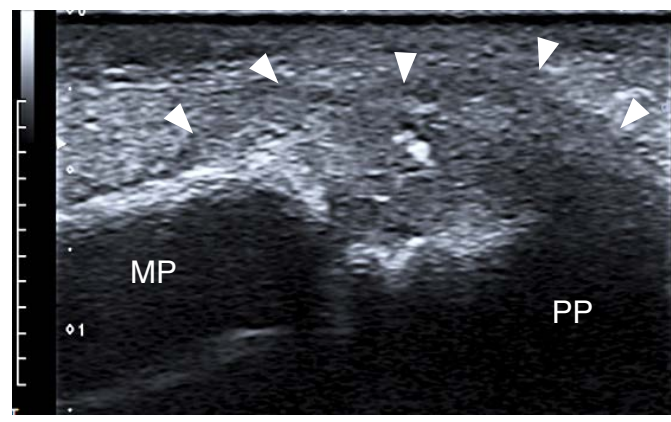

D

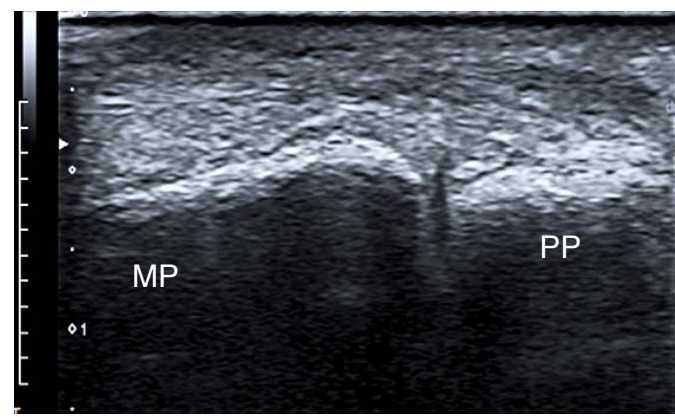

$\mathrm{E}$

show an irregular, swollen, and hypoechoic ulnar collateral ligament (arrowheads in

D) in comparison with a normal radial collateral ligament (E). MP, middle phalanx; PP, proximal phalanx. 


\section{Lateral Aspect}

\section{Normal Anatomy}

The main stabilizers of the MCP and PIP joints include the volar plate and collateral ligaments (Fig. 20). The collateral ligaments of the MCP joint are taut during flexion and lax during extension, allowing abduction and adduction [9]. The collateral ligament of the PIP joints consists of the proper and the accessory collateral ligaments; the proper collateral ligament is taut during flexion, whereas the accessory collateral ligament is taut during extension [4]. The collateral ligaments of the IP joints can be examined in both transverse and longitudinal US scans. However, visualization of the collateral ligaments of the MCP joint is limited to longitudinal access, except at the level of the thumb and at the radial aspect of the second MCP and ulnar aspect of the fifth MCP joint [2]. On US, the collateral ligaments are seen as anisotropic bands crossing the joint and inserting into the boundaries of adjacent bones. Both hyperechoic and hypoechoic appearances may be possible, depending on the US beam direction (Figs. 21, 22) [2].

The MCP joint of the thumb is stabilized by the volar plate, collateral ligaments (similar to the PIP and MCP joints of other fingers), and additional musculotendinous structures. The adductor

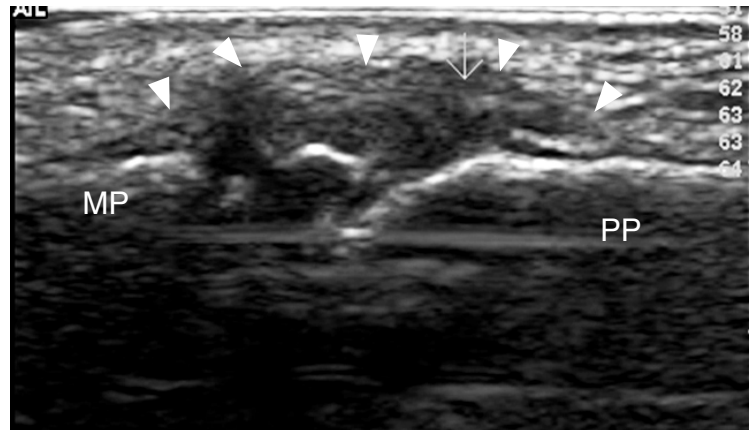

A

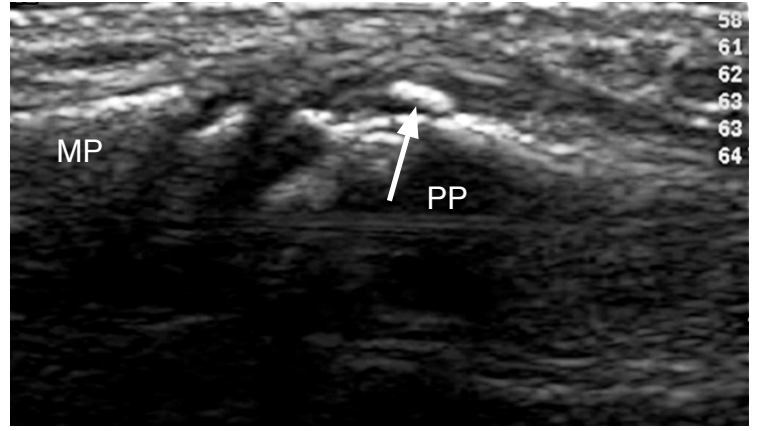

B

Fig. 22. Collateral ligament injury of the proximal interphalangeal (PIP) joint in a 9-year-old man.

A. Longitudinal sonogram shows an irregular, swollen, and hypoechoic radial collateral ligament (arrowheads) of the fourth PIP joint. B. Note the small hyperechoic fragment (arrow) corresponding to the cortical avulsion of the proximal phalangeal head on another longitudinal sonogram. MP, middle phalanx; PP, proximal phalanx.

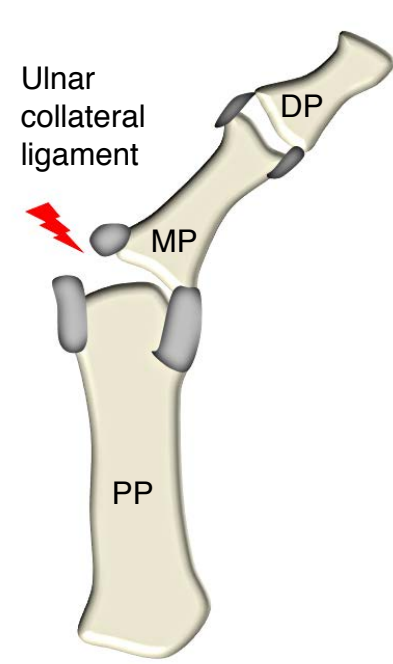

A

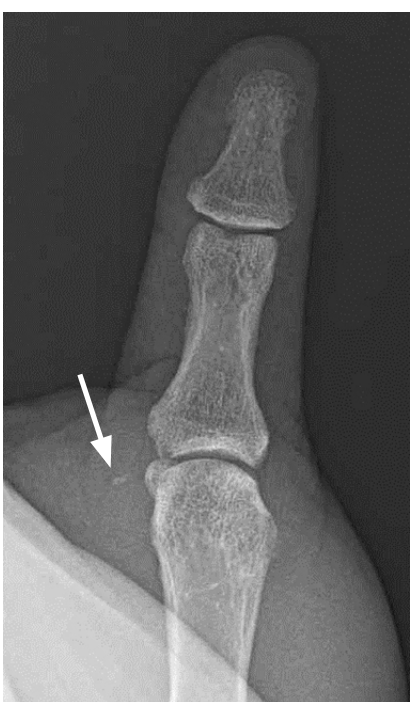

B

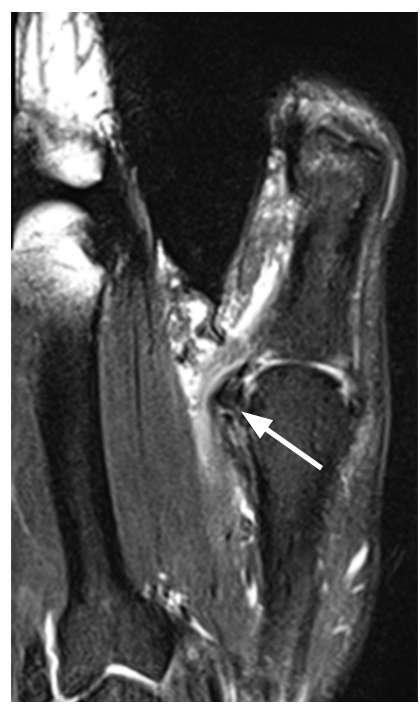

C

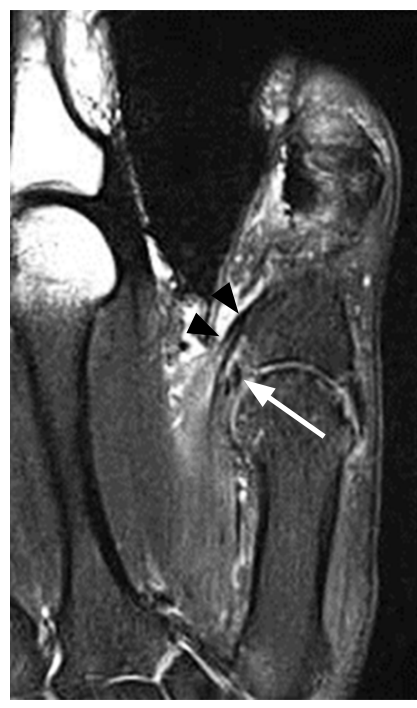

D

Fig. 23. Gamekeeper's thumb in a 32-year-old man.

A, B. In a ulnar collateral ligament injury called gamekeeper's thumb (A), radiograph shows a tiny bone fragment (arrow) adjacent to the first metacarpophalangeal joint on the ulnar side (B). C, D. A nondisplaced torn ulnar collateral ligament (arrows) appears as a gap under the adductor aponeurosis (arrowheads) on coronal T2 fat saturation images. DP, distal phalanx; MP, middle phalanx; PP, proximal phalanx. 
pollicis has a strong tendinous point of insertion into the PP and the volar plate/sesamoid complex. Some of its fibers also contribute to the adductor aponeurosis, which covers the ulnar collateral ligament (UCL) of the MCP joint of the thumb [4]. The adductor aponeurosis can be distinguished as a thin band overlying the UCL on US using high-frequency transducers [2].

\section{UCL Injuries (Gamekeeper's Thumb, Stener Lesions)}

UCL injuries were originally described in gamekeepers who frequently strangled rabbits, and recently in skiers due to the way that they grip the ski pole during a fall [10]. The mechanism of UCL injury involves hyperabduction of the MCP joint with varying degrees of hyperextension $[10,11]$. The ligament is usually torn at its distal insertion site (Fig. 23), and the UCL can remain in its anatomical position, deep in the adductor aponeurosis (Fig. 24A), or can be displaced to the proximal edge of the adductor aponeurosis (Fig. 24B, C), depending on the force exerted during the injury [10]. Although nondisplaced UCL injuries (gamekeeper's thumb) can be treated without surgery, displaced UCL injuries (Stener lesions) require surgical repair [10]. Therefore, it is clinically important to differentiate between nondisplaced and displaced ruptures of the UCL.

According to Bianchi and Martinoli [2], a partially torn UCL appears thickened and hypoechoic on US due to intrasubstantial edema and hemorrhage. On US of complete nondisplaced ruptures, the end of the UCL can be identified over the proximal or distal

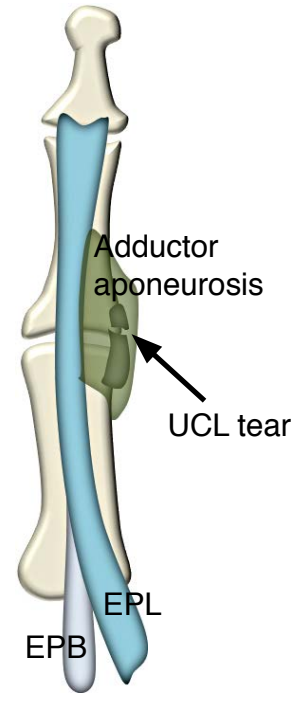

A

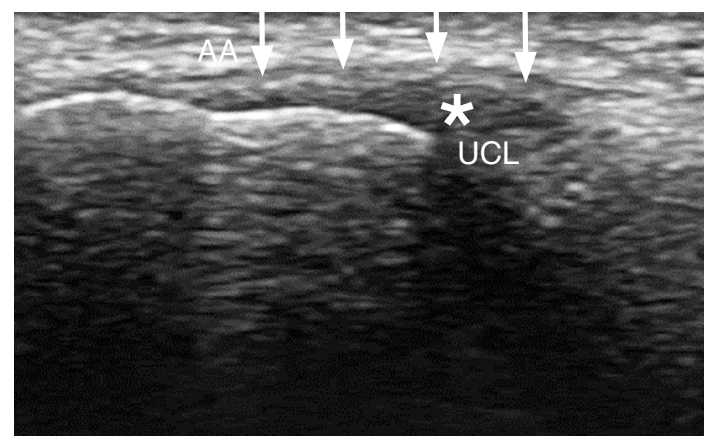

A

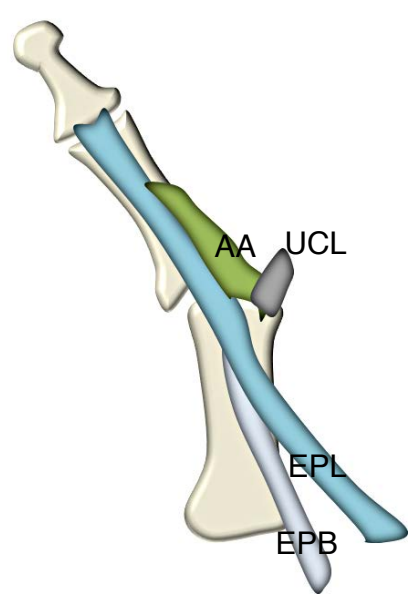

B

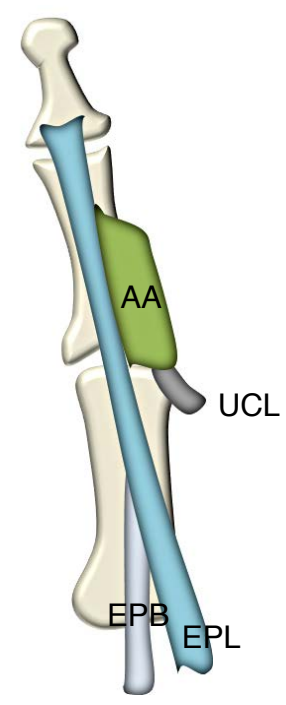

C
Fig. 24. Schematic images of Stener lesions.

A-C. Different from gamekeeper's thumb with ulnar collateral ligament (UCL) remaining in its anatomical position (A), Stener lesions $(B, C)$ show the torn end of the UCL displaced superficially to the adductor pollicis aponeurosis (AA). EPB, extensor pollicis brevis; $E P L$, extensor pollicis longus.

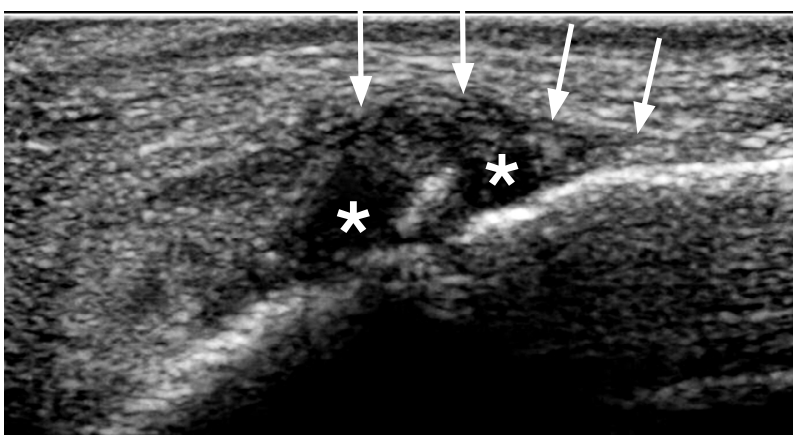

B

Fig. 25. Gamekeeper's thumb in a 41-year-old man.

A. Longitudinal sonogram of a normal thumb shows a normal ulnar collateral ligament (UCL, asterisk). The deeper fibers of the UCL appear anisotropic. Superficially, it is covered by an additional thin, hyperechoic band corresponding to the adductor pollicis aponeurosis (AA, arrows), which is variably visualized in normal fingers. B. Longitudinal sonogram shows the UCL tear (asterisks) as an irregularly swollen, hypoechoic structure under the AA (arrows). No significant ligament retraction is visible. 
insertion of the ligament, but it remains in its anatomic position (Fig. 25). Displaced ruptures of the UCL are typically seen as a round hypoechoic mass proximal to the joint and at the level of the metacarpal neck. This mass corresponds to the displaced and retracted end of the ligament over the adductor aponeurosis, forming the ball of the so-called yo-yo sign. In uncertain cases, a dynamic study using radial stress can be helpful. However, since ligament displacement can be caused by excessive force, only gentle and careful stress should be applied [2].

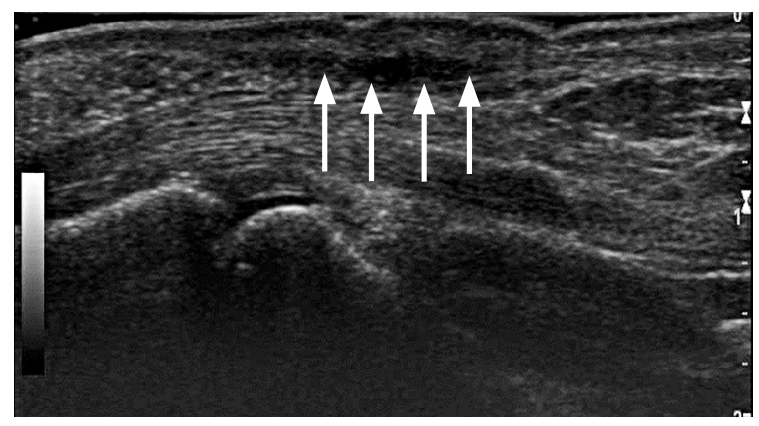

A

\section{Other Conditions}

\section{Dupuytren Disease}

Dupuytren disease (palmar fibromatosis) is the most common type of fibromatosis. It occurs in approximately $1 \%-2 \%$ of the general population and may present bilaterally in $42 \%-60 \%$ of cases [12]. It is characterized by subcutaneous nodules and variable retraction of the palmar aponeurosis, resulting in limited movement of one or more fingers [12]. In Dupuytren disease, the more commonly affected site is the ulnar aspect of the fascia near the distal crease of

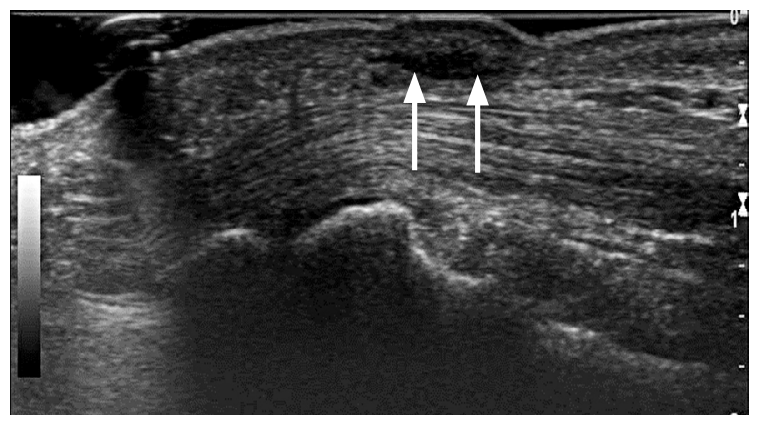

B

Fig. 26. Dupuytren disease in a 53-year-old man.

A, B. Longitudinal sonograms demonstrate fusiform hypoechoic thickening (arrows) of the palmar aponeurosis, resembling soft-tissue masses. These hypoechoic lesions (arrows) are separated from the flexor tendons by a thin layer of hyperechoic connective tissue.

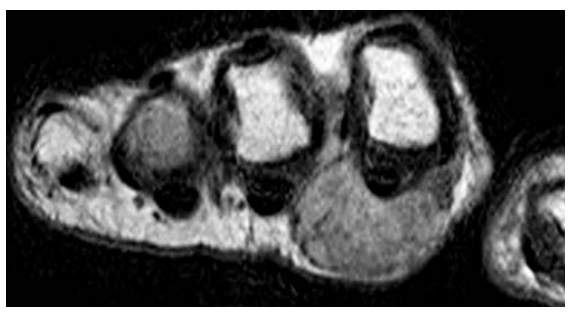

A

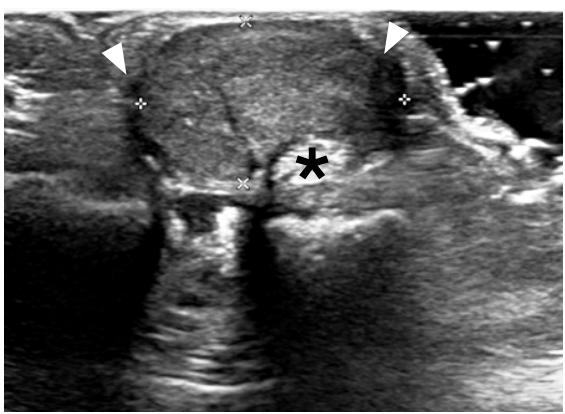

C

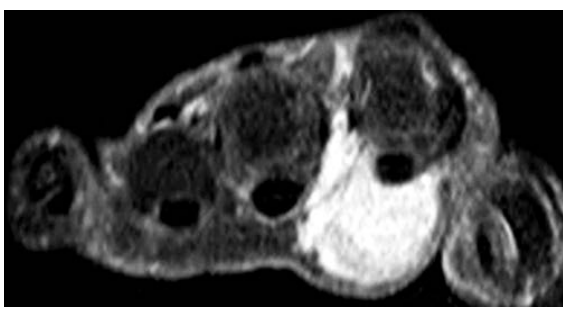

B

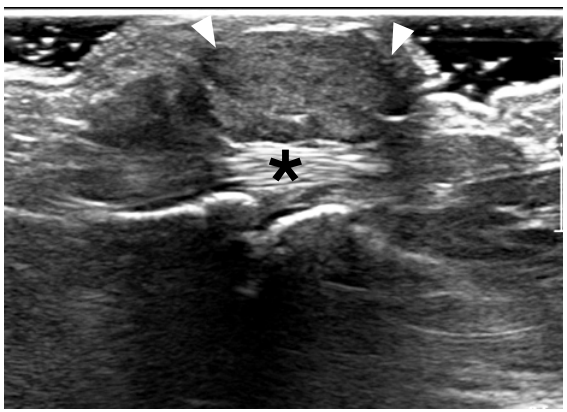

D

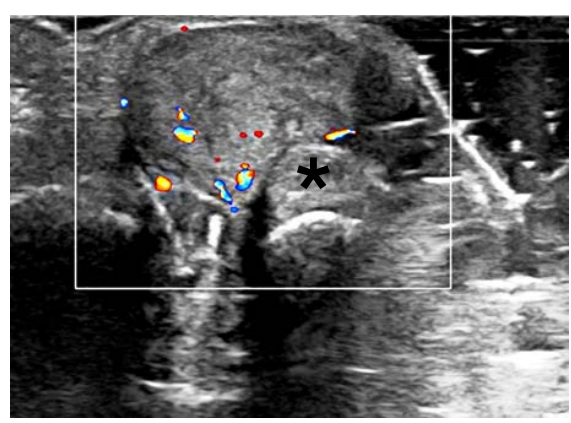

$\mathrm{E}$

Fig. 27. Tenosynovial giant cell tumor in a 76-year-old woman.

A, B. Axial T1-weighted magnetic resonance (MR) image (A) and coronal T2 fat saturation MR image (B) show a well-defined soft tissue mass on the volar aspect of the second finger. C, D. Transverse (C) and longitudinal (D) sonograms demonstrate a lobulated, well-marginated isoechoic to hypoechoic mass (arrowheads) located close to the flexor tendon (asterisk). E. Color Doppler ultrasonography image reveals focal vascularity inside the tumor (asterisk, the flexor tendon). 


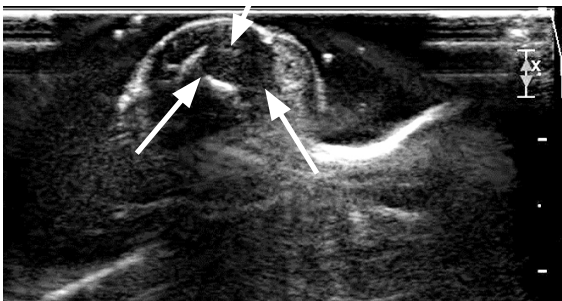

A

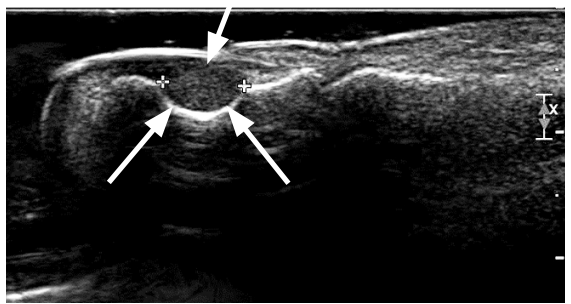

B

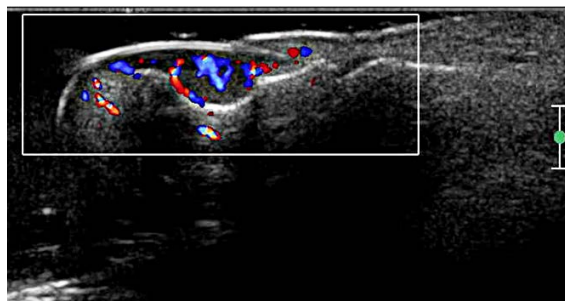

c

Fig. 28. Subungual glomus tumor in a 50-year-old man.

A, B. Transverse sonogram (A) and a longitudinal sonogram (B) reveal a solid hypoechoic mass (arrows) beneath the nail. Note the pressure erosion of the underlying bony cortex. C. Marked intratumoral vascularity is seen on color Doppler ultrasonography.

the hand and the bases of the fourth and fifth fingers, followed by the second and third fingers [12]. This lesion is visualized on US as a nodular thickening of the palmar aponeurosis with sharp margins and hypoechogenicity between the skin and the flexor tendons (Fig. 26) [13]. These nodules show no significant internal vascularity on color Doppler US [13]. In dynamic studies, palmar fibromatosis shows adhesions with the flexor tendon during finger movements $[2,13]$.

\section{Ganglion Cysts}

The most common space-occupying lesions of the hand and fingers are ganglion cysts [14], which are composed of mucoid material surrounded by fibrous tissue without a synovial lining and have an unclear etiology [15]. Digital ganglion cysts present as small, hard masses on the volar aspect of the finger and can cause significant discomfort and pain [2]. US depicts these cysts as small anechoic lesions adjacent to IP joints or tendon sheaths, with thin walls and posterior acoustic enhancement [2]. They usually extend along the lateral and medial aspects of the flexor tendons. In dynamic studies, US demonstrates smooth gliding of the flexor tendons without a change in the size or position of the ganglion cyst itself [2].

\section{Tenosynovial Giant Cell Tumors}

Tenosynovial giant cell tumors, which are more commonly referred to as giant cell tumors of the tendon sheath or as a localized form of pigmented villonodular synovitis, are the second most common soft-tissue mass of the hand and finger [2]. It is a slow-growing solid mass with hypoechogenicity and sharp margins at the palmar aspect of the fingers and is typically located on the adjacent normal flexor tendons [2]. In contrast to digital ganglion cysts, tenosynovial giant cell tumors show internal echoes and lack posterior acoustic enhancement on US. Cortical pressure erosion has been reported in $10 \%-50 \%$ of cases, and eccentric displacement of the digital arteries can generally be observed [2]. On color Doppler US, these lesions usually demonstrate poor internal vasculature. Although the
US findings for this tumor are not specific, the typical presence of a mass adjacent to the tendon sheath with the above findings is strongly suggestive of a tenosynovial giant cell tumor (Fig. 27) $[2,16]$.

\section{Glomus Tumors}

Glomus tumors are rare benign tumors that arise from the neuromyoarterial glomus, which is located beneath the nail or over the palmar aspect of the fingertip $[2,17]$. Typical symptoms of a glomus tumor are excruciating pain and temperature sensitivity [17]. On US, these tumors appear as a small, solid mass beneath the nail with homogeneous hypoechogenicity, and they may be associated with bone erosion of the underlying phalanx $[2,17]$. Moreover, the detection of small subungual lesions can be difficult due to US artifacts caused by the curvature of the nail plate and the lateral nail folds [17]. Color and power Doppler US show this tumor as a hypervascular mass due to the high-velocity flow of intratumoral shunt vessels $[18,19]$. This is a reasonably specific finding for the diagnosis of a glomus tumor. Although no specific US findings can be used to differentiate a glomus tumor from other soft tissue tumors, the combination of the appropriate clinical symptoms, such as excruciating pain, and US findings, such as the typical subungual location and hypervascularity, can be used to diagnose a glomus tumor (Fig. 28) [17].

\section{Conclusion}

Although the finger is a small and complex structure that can be difficult to evaluate using imaging modalities, the recent development of high-frequency transducers has made US a valuable tool for evaluating the normal anatomy and various pathologies of the finger. US is fast and easy to perform and provides dynamic assessment of the flexor and extensor tendons, collateral ligaments, and supporting structures of the finger, such as the extensor hood and the volar plate, as well as space-occupying lesions. 
ORCID: Seun Ah Lee: http://orcid.org/0000-0002-6190-5503; Baek Hyun Kim: http://orcid.org/0000-0002-3284-1803; Seon-Jeong Kim: http://orcid.org/00000001-9064-7155; Ji Na Kim: http://orcid.org/0000-0002-1877-9273; Sun-Young Park: http://orcid.org/0000-0001-6500-3411; Kyunghee Choi: http://orcid.org/00000003-1287-6379

\section{Conflict of Interest}

No potential conflict of interest relevant to this article was reported.

\section{Supplementary Material}

Video clip 1. Sagittal band injury. Since the sagittal band prevents deviation of the extnesor tendon during metacarpophalangeal joint flexion and bowstring during hyperextension, sagittal band injury causes extensor tendon dislocation during flexion (http://dx.doi. org/10.14366/usg.15051.v001).

Video clip 2. Ultrasonography of the flexor tendon. If the examiner moves the distal phalanx only, while locking the middle phalanx, the flexor digitorum profundus tendon can be seen to glide under a stationary flexor digitorum superficialis tendon (http://dx.doi. org/10.14366/usg.15051.v002).

Video clip 3. Trigger finger. Dynamic ultrasonography can visualize the locking and snapping of the flexor tendon at the metacarpophalangeal joint level (http://dx.doi.org/10.14366/ usg.15051.v003).

\section{References}

1. McNally EG. Ultrasound of the small joints of the hands and feet: current status. Skeletal Radiol 2008;37:99-113.

2. Bianchi S, Martinoli C. Ultrasound of the musculoskeletal system. New York: Springer, 2007.

3. Lee JC, Healy JC. Normal sonographic anatomy of the wrist and hand. Radiographics 2005;25:1577-1590.

4. Clavero JA, Alomar X, Monill JM, Esplugas M, Golano P, Mendoza $M$, et al. MR imaging of ligament and tendon injuries of the fingers. Radiographics 2002;22:237-256.

5. Willekens I, Kichouh M, Boulet C, De Maeseneer M, Clarys JP, de Mey J. Ultrasound follow-up of posttraumatic injuries of the sagittal band of the dorsal hood treated by a conservative approach. Eur J Radiol 2015;84:278-283.

6. Wheeldon FT. Recurrent dislocation of extensor tendons in the hand. J Bone Joint Surg Br 1954;36:612-617.

7. Boutry N, Titecat M, Demondion X, Glaude E, Fontaine C, Cotten A. High-frequency ultrasonographic examination of the finger pulley system. J Ultrasound Med 2005;24:1333-1339.

8. Guerini H, Pessis E, Theumann N, Le Quintrec JS, Campagna R, Chevrot $A$, et al. Sonographic appearance of trigger fingers. J Ultrasound Med 2008;27:1407-1413.

9. Masson JA, Golimbu CN, Grossman JA. MR imaging of the metacarpophalangeal joints. Magn Reson Imaging Clin N Am 1995:3:313-325.

10. Hergan K, Mittler C, Oser W. Pitfalls in sonography of the Gamekeeper's thumb. Eur Radiol 1997;7:65-69.

11. Ebrahim FS, De Maeseneer $M$, Jager $T$, Marcelis $S$, Jamadar DA, Jacobson JA. US diagnosis of UCL tears of the thumb and Stener lesions: technique, pattern-based approach, and differential diagnosis. Radiographics 2006;26:1007-1020.

12. Robbin MR, Murphey MD, Temple HT, Kransdorf MJ, Choi JJ. Imaging of musculoskeletal fibromatosis. Radiographics 2001:21:585-600.

13. Markham DE, Wood MR. Ultrasound for Dupuytren's contracture Physiotherapy 1980;66:55-58.

14. Garcia J, Bianchi S. Diagnostic imaging of tumors of the hand and wrist. Eur Radiol 2001;11:1470-1482.

15. Cardinal E, Buckwalter KA, Braunstein EM, Mih AD. Occult dorsal carpal ganglion: comparison of US and MR imaging. Radiology 1994; 193:259-262.

16. Horcajadas AB, Lafuente $\mathrm{JL}$, de la Cruz Burgos R, Muniz SH, Roca $S A$, Ortega $S G$, et al. Ultrasound and MR findings in tumor and tumor-like lesions of the fingers. Eur Radiol 2003;13:672-685.

17. Fornage BD. Glomus tumors in the fingers: diagnosis with US. Radiology 1988;167:183-185.

18. Bianchi S, Della Santa D, Glauser T, Beaulieu JY, van Aaken J. Sonography of masses of the wrist and hand. AJR Am J Roentgenol 2008:191:1767-1775

19. Reynolds DL Jr, Jacobson JA, Inampudi P, Jamadar DA, Ebrahim FS, Hayes CW. Sonographic characteristics of peripheral nerve sheath tumors. AJR Am J Roentgenol 2004;182:741-744. 